\title{
The Strategic Planning and Branding Tactics of Meituan
}

\author{
Chuhe Xuan * \\ School of Arts and Science, University of Virginia, Charlosville, USA \\ *cx8wm@virginia.edu
}

\begin{abstract}
Meituan, an Internet company that focuses on businesses such as food delivery and hotelordering, was first launched in 2009. Within a decade, Meituan has evolved into a shopping platform that Chinese rely on, due to Meituan's smart strategic planning, marketing positioning, high working efficiency, and its variety of business branches. Meituan has emerged as one of the Top 20 Risers global brands and ranked 54th among the Top 100 brands in 2020. As a service company that occupies the biggest portion of food delivery within the Chinese market, it's of significance to explore what factors make Meituan the most successful and popular platform that changes people's way of living. By investigating on Meituan's financial reports, industry analysis, case studies and peer brand comparison, this paper will analyze Meituan's strategic planning and development of its business and its linkage with consumer psychology, examine the factors that contribute to Meituan's success, and provide inspiration of the brand's future development.
\end{abstract}

Keywords: Meituan; Food Delivery; Color; Logo; Brand Positioning; Strategic Planning.

\section{Introduction}

Meituan, a leading innovative App for local life services, has been launched for 10 years. According to the report, Meituan owns 142 billion USD market capitalization as for July 17, 2020, and its performance after its IPO in 2018 is striking, as shown in Figure 1. Meituan starts its business from collective buying, and soon expands its business to food delivery and food review, and then initiate other businesses such as online ticketing, travel, wine, fresh-food supermarket, etc. A year after Meituan was launched, there were already 5,000 group-buying wesbites, and companies were facing fierce competition in the race of market share [2]. Meituan adopts the aggressive expansion strategy, as can be observed from its wide range of business and expansion from major cities to small cities [2]. With its strategic planning, corporate management, and integrated marketing strategies, Meituan's success and dominance position seem to be inevitable. Until 2019, Meituan has become the largest food-delivery provider in China that dominates $65.1 \%$, while its biggest competitor "Ele Me" only dominates 27.4\% [2]. Therefore, Meituan's tremendous, successful expansion within the decade is a good business model for researchers to study on and further explore.

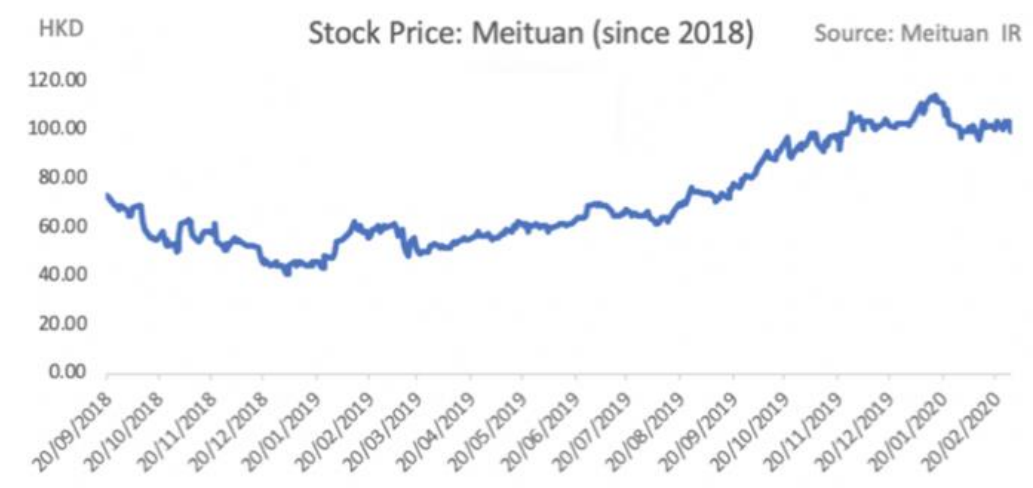

Figure 1. Meituan's Stock Price Since 2018 


\section{Meituan is the largest food-delivery service provider in China}

Market share, Q2 2019

- Ele.me Star Select $5.4 \%$

Other

$2.1 \%$

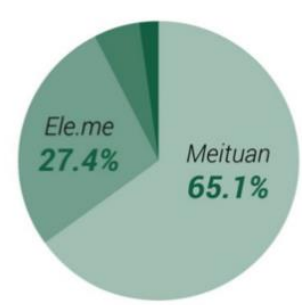

Figure 2. Meituan's Market Share on Food-delivery Service in 2019

\section{Meituan's Strategic Planning}

Meituan is under rapid development after launching the business in 2010, especially when it expands its business to not only food delivery (Meituan App), food review (Dazhong Dianping) and hotel-ordering (Meituan App), but also living entertainment, such as online car-ordering and fresh food chain supermarkets. Many people are concerned about the track and future of Meituan's tremendous expansion within the decade. However, it is essential for us to look at Meituan's strategic planning in a broader perspective, because only if we put Meituan's development into the whole picture, we could understand the underlying logic of Meituan's wide variety of expansion. Meituan develops many strategic plans, displaying its wisdom in business operation.

\subsection{The "Super Platform" Strategy}

Unlike the business like Didi Chuxing (“滴滴出行” in Chinese) that develops its taxi service with deep, vertical approach, Meituan applies the "super platform" strategy. A "super platform" usually refers to apps that have absolutely large user scale and a high frequency of use that can achieve a closed-loop consumption of monopolizing user minds (monopoly traffic pools) and to master dominance of user traffic. The most important significance of becoming a super platform is that the platform becomes the absolute leader of traffic distribution, and in the Internet era, traffic is the most important resource (users, flow, monetization, and profit are expected to follow).

In the mobile Internet era, the threshold for jumping between apps is high. To use a new app requires users to download and then register, which is burdensome and causes users to be more inclined to solve all their needs in one app, so the super platform is born. Only a few apps, those most useful and convenient ones, can last for a long time on a user's mobile phone. Therefore, the super platform is becoming an increasingly important traffic distribution portal and holds the power of traffic distribution in the mobile Internet era. Meituan wisely catches consumer behaviors and psychology, and develop the brand with the "super platform" strategy.

Even though Meituan's business mainly focuses on food-delivery service, Meituan's ultimate goal is to establish a "Super Platform" within the context of local life services and fulfills users' wide variety of living needs. Until now, Meituan has developed several chains of services: dining services such as food delivery and in-store dining, traveling branch such as hotel-ordering and ticket-ordering, transportation aspect like Meituan bike and car-ordering, entertainment branch like hair-cutting and filming, and retail services that enables the users to order fresh food. The variety of services Meituan provides within the platform takes local life services as a large-scale closed-loop scenario. By meeting the different needs of the same type of users, Meituan earns consumer's higher loyalty of its brand. If consumers are loyal to one of Meituan's businesses, such as Meituan's food delivery service, they tend to choose the other services of Meituan rather than choosing to use services provided by other companies. Because all the services of Meituan are centered in the ideal of bringing the users with higher-quality, more convenient services to improve the local living experience. The ultimate goal of Meituan's super platform strategy is to control the traffic under the context of living, which internally could inflow a wider range of consumers and new services, and externally could achieve dimensional reduction attack to the competitors that only have single-dimension business. The wide range of 
services of Meituan increases its credibility within the industry, especially compared to its competitors like "Ele Me" and "Baidu Food Delivery," which focus exclusively on food delivery service but fail to fulfill users' needs in other aspects of living.

\subsection{The "Supply-Side" Reform}

Meituan's second strategy to promote its development is the "supply-side reform." With Internet and smart phones becoming more and more accessible in China, Meituan has started on reforming the layout of its supply-side. Meituan intends to probe into the industry chain and uses its operation experience of the downstream, the understanding of the industry and the technical advantages to bring revolution to the upstream of the industry, and achieve the goal of increasing the value of the industry chain and reinforce the barriers of the industry. To be more specific, Meituan provides B-end merchants with supply-side solutions covering every link from preparation to operation, from the front-end to the back-end. While improving the efficiency of the supply-side, it also raises the industry's entry barriers and strengthens its own barriers; at the same time, B-side Merchants pay for efficiency improvements, increasing the value of Meituan in the industry chain. Initially, when all the businesses were under rough-cut operations, new businesses can survive under the set of rules of operations. After Meituan initiated the supply-side reform, the businesses that accepted supply-side reforms improved their efficiency and reduced costs, while the businesses that did not accept supplyside reforms encountered a major problem of high costs and were eliminated as a result. Since then, supply-side reforms have become a prerequisite for merchants to run stores, and because this set of supply-side reform solutions is based on the Meituan platform, Meituan's control over the B-side will be greatly improved. After taking control of the B-side, Meituan can use the B-side barriers to attack its competitors, cut off the possibility of new competitors to enter the market, thereby greatly enhancing its own power and barrier [3].

\subsection{The Logic of Business Expansion of Meituan}

After understanding the two major strategies of Meituan, we can have a better understanding of the logic of Meituan's expansion. For example, businesses like bike-sharing, online ride-hailing, grocery shopping and consumer loans are based on the super-platform strategy, while Kuailu, ERP, aggregate payment, and merchant loans are all built on the supply-side strategy. Therefore, Meituan's future business expansion is foreseeable: if it is in line with the strategic value, Meituan will invest in the program even if it may cause loss of money, such as Meituan's bike-sharing service; otherwise, Meituan will be inclined to give up if the program could bring short-term earnings but fail to align with Meituan's strategic value. The significance of strategic synergy is that it allows different businesses to be put together to achieve the effect of $1+1>2$. This is an important advantage of Meituan over other single-business companies: it can make the industry work where the singlebusiness model cannot.

\section{Meituan's Core Competitiveness}

Except for Meituan's profound strategic planning and organization, Meituan has at least four core competitiveness, compared to the other online apps that focus on life-quality improvement, including its intangible assets, capital advantages, and talent advantages.

The first competitiveness is Meituan's intangible assets. Intangible assets are divided into brand advantages and technical advantages: as for the brand advantages, the competiveness of the brand is reflected in the fact that Meituan has monopolized the user-perceptions and has attracted a big group of loyal users, and it is very difficult for latecomers to change this perception; technical advantages come from the continuous revisions and improvements built upon huge research and development (R\&D) investment and the tremendous number of orders. Meituan's R\&D expenditure over the years has totaled nearly 30 billion yuan, and the order volume is nearly 40 billion [4]. Even though 
latecomers could also invest huge amounts in research and development, it is difficult to do so without sufficient sample size (i.e, a great number of users).

The second competitiveness of Meituan is its capital advantages. Meituan has more than 50 billion RMB of funds in its account, and it already has the ability to produce large-scale self-sustainability. When the latecomers enter, Meituan can use its absolute capital advantage to compete with the latecomers, and the latecomers will be defeated before they could reach a scale [5].

At last, Meituan also make a great effort in employing talents. From the perspective of market value, Meituan is already the third largest Internet enterprises in China. Larger platform, like Meituan, can attract better talents and teams, including the recruitment of executives or new graduates, while smaller-size enterprises can only find sub-optimal or even ordinary talents and teams.

\section{Meituan's Strategic Vision and Management Layout}

What contributes to Meituan overperforming the thousands of competitors and ultimately becoming the lead of the market are its excellent strategic ability and strong management capacity. Strategic ability enables Meituan to correctly spend its resources in the right places, while organizational capacity enables the strategy effectively to land and implement successfully at the from the base level to the top. The success of an enterprise requires its strategic ability and its organizational ability. Strategic leadership, coupled with organizational ability, have led to Meituan's breakthrough: the market share at the end of 2011 jumped to the top of the market, and since then, Meituan becomes the absolute market leader, and the gap between Meituan its competitors have been widening.

\subsection{Meituan's Direction of Entrepreneurship}

Meituan's direction of entrepreneurship is based on the "four verticals and three horizontals" theory [6], put forward in 2009 by Xing Wang, the founder of Meituan. The "three horizontals" refers to the three Internet technology's changing direction, including searching function, socializing function, and mobility function, and the "four verticals" refers to the development of four user needs, including information-collection, communication, entertainment, and business. At the intersection of every technology and user needs, there are significant entrepreneurial opportunities, but the only area that had yet been developed was group purchase, the area that Meituan had set its focus on.

\subsection{Meituan's Focus on Business}

In addition, Meituan's direction of its business is clear: Meituan only focus on service-focused group purchase. At the start of group purchase industry, because of the high gross-profit of the physical group purchase, many enterprises put their emphasis on that, which in turn causes such business to bloom. [7] According to Wang, physical group purchase requires a clear experience advantage on its product selection, price, logistics and other aspects, otherwise it certainly cannot compete against Taobao given its business scale. Therefore, Meituan adheres to the service-focused group purchase, and the proportion of physical group purchase is controlled below $10 \%$ [5].

\subsection{Meituan's Operation Philosophy}

Meituan also adheres to the principle that "Consumers come the first and businesses come the second," reflecting Meituan's deep understanding of the nature of group purchase business. At that time, Groupon, the originator of group purchase business, believed that merchants were its direct customers, so its philosophy puts merchants as their priority and consumers' experience come the second. Meituan, however, believed that group purchase relies on businesses, but business cannot live without consumers. This principle of putting consumers as priority has played an important role in the decision-making of many key issues, so that Meituan continues to accumulate consumers' favor and positive impression, and that is a core reason that Meituan could achieve its huge expansion on group purchase. 
From the decision-making philosophy and layout of the Meituan's expansion, we see its excellent strategic ability. Every step is the result of careful consideration, from determining the direction of entrepreneurship, establishing a business model, to determining its clear business direction and development priorities, and all of the decisions indicate Meituan's excellent insight, judgment, and strategic vision, enabling Meituan to constantly make right choices, accumulate small wins to big wins and ultimately lead Chinese market in the field of group purchasing [9].

\section{Meituan's Branding Strategies}

Except for the excellent strategic vision, planning and high-quality service, Meituan has many marketing strategies that effectively attract consumers' eyes, including Meituan's logo, brand positioning, the use of color, and advertising channels and platforms. All the elements contribute to establish Meituan's brand value with high consistency.

\subsection{Meituan's Alternation of Logo and Color}

Ever since Meituan was launched in 2010, Meituan's logo has made a few changes on the shape, typeface, and color, in order to cater to the changing consumer preferences and market needs. According to Jamie, redesigning the logo is an important step toward creating an up-to-date image and impression toward the company [3], but meanwhile, the logo should not have constant revolutionary changes because it breaks the brand consistency. A successful brand re-design can lead to more sales, a better impression and a better align with brand values. Just as Figure 3 shows, the main colors of Meituan's old logo are white and green, and it evolves into a combination of black, white and yellow. Yellow represents youth, vitality, and warmth, and makes people relate to hunger, catering to Meituan's main business of food delivery and in-door food service that should associate with vigor.

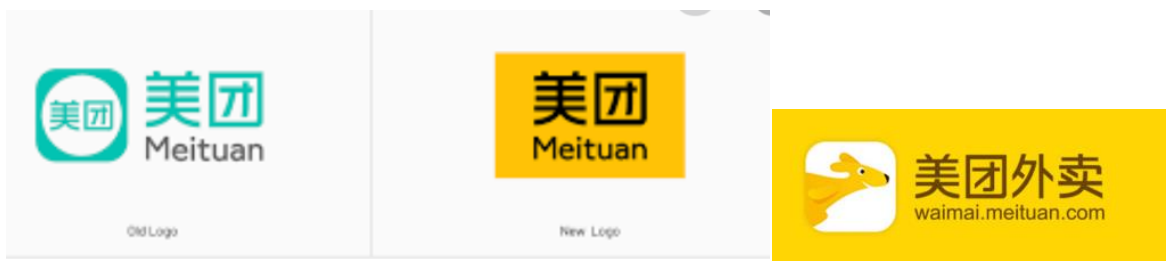

Figure 3. Meituan's changes of logo

\subsection{Brand Positioning}

Meituan's food delivery service serves as a good alternation of family-cooking and in-store dining, aiming to save the users' time and offer another lifestyle that is easier and more convenient. Meituan devotes to offer a wide variety of choices: it has 6,200,000 merchants, covering 2800 cities in China, and owns 3,990,000 riders (delivery people) [4]. Addition to that, due to its "super-platform strategy," Meituan aims to establish a platform that satisfies a variety of daily needs, from food delivery, carordering to hotel and ticket's ordering, so Meituan positions to the people of different age groups (but especially the younger generation), depending on their personal needs. Therefore, we could summarize Meituan's brand positioning statement into a sentence: to people who would like to adapt to an easier, more highly efficient and comfortable lifestyle with only one click, Meituan is one of the few platforms that satisfies needs of living, because Meituan covers the largest number of restaurants, the widest coverage of cities and the highest number of riders who processes delivery with a high efficiency and a short delivery time [10].

\subsection{Advertising Channels}

Meituan uses different advertising channels to help build its brand value and reinforce the brand popularity. The advertising effectiveness of Meituan remains high, generating an average of \$45.9 per ad, far more than $\$ 5$ for a single line-of-business company in 2019 [5]. The main channels of 
advertisement are a combination of online and offline. Online channels include Weibo, Wechat, and Meituan APP, and just as Figure 4 shows, the color of the advertisement design and the Meituan official APP also use yellow and white as its primary colors, aligning with Meituan's logo, and the wide use of color, bright yellow, help builds Meituan's memory point, just as the color of mint green is always associate with the jewelry brand Tiffany. On the other hand, Meituan's offline advertisement is via Meituan's riders. In most of China's cities, we can see Meituan riders' typical clothing: a distinctive yellow sweater with the character “Meituan" (“美团” in Chinese) on it, just as Figure 5 shows. The large number of riders walking on the streets is a strong way of advertisement, because it shows Meituan's popularity [8].

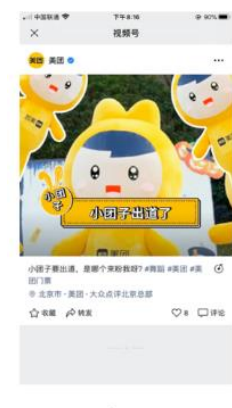

Wechat

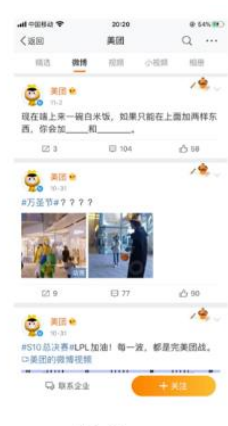

Weibo

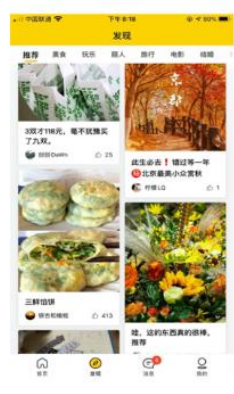

Meituan App

Figure 4. Meituan's online advising channels

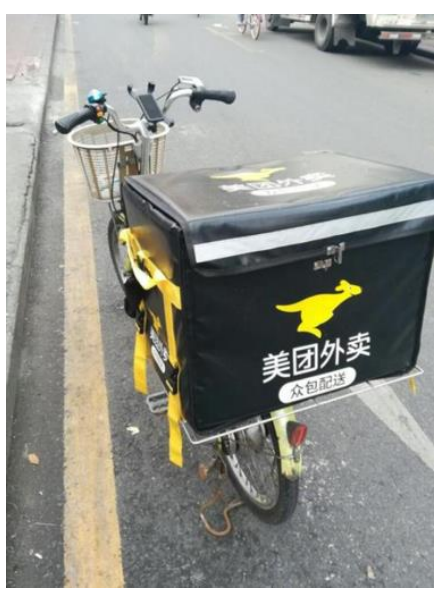

Figure 5. Meituan riders on the street

\section{Conclusion}

In sum, the success of Meituan depends on its strategic vision, long-term insight, plannable layout of management, decisive judgement, and business capacity, and also its proficient grasp on marketing strategies, such as colors, logo, consumer psychology, and brand positioning, which all contributes to the fact that today Meituan dominates in Chinese market, especially for group purchase and fooddelivery service.

Meituan's success could potentially be a good model for other corporates, whatever their businesses are, to learn from. Until now, Meituan's latest brand value is $\$ 23,911$ million and ranks $54^{\text {th }}$ of the Top 100 global business. The emergence of Meituan provides an e-commerce platform to change people's way of living by reducing their live costs, saving their time from cooking and improving their living quality by the means of technology. This innovative, people-centered business philosophy is the core of its secret of success. 


\section{References}

[1] Meituan: BrandZ, www.brandz.com/brand/11227. Accessed 23 September 2020.

[2] Lin, Lingyi. "After Establishing Meituan as the Biggest on-demand Service Platform in China, Hidden Hero Wang Xing Retires,” KrASIA, July 15, 2020. https://kr-asia.com/after-establishing-meituan-as-thebiggest-on-demand-service-platform-in-china-hidden-hero-wang-huiwen-retires.

[3] Liu, Coco. "Meituan's Sales Surge Alongside China's Appetite for Takeout," Bloomberg Technology, Nov 30, 2020. https://www.bloomberg.com/news/articles/2020-11-30/meituan-s-sales-surge-alongsidechina-s-appetite-for-takeout.

[4] Jamie. "The Effects of Changing Your Logo Design", Branding, Creativity \& Inspiration, Importance of Graphic Design, News, Tips and Tricks. April 20, 2014.

[5] "Meituan-Dianping's Corporate Research Analysis," Industry Research Review, November 28, 2020. https://blog.csdn.net/qianchou2/article/details/110295095.

[6] "Meituan's Review on Strategies, Planning and Capacity," Guosheng Securities Report, May 21, 2020.

[7] Liu, Yue. "Meituan-Dianping Research Report," Equal Ocean Intelligence. Aug 27, 2020. https:// equalocean.com/research/2020082714602.

[8] Md. Saikat Hosen. "O2O Business Model of Meituan in China," Global Disclosure of Economics and Business, Volumn 9, Aug 2020. https:// www. research gate. net/ publication/ 343788746 _O2O _ Business_Model_of_Meituan_in_China.

[9] Liu, Yujing. "Budweiser, Anta Sports and Meituan join Hang Seng Index, displacing Swire Pacific from Hong Kong's stock benchmark," Yahoo! Finance. Nov 13, 2020. https:// finance. yahoo. com/ news/ Budweiser-anta-sports-food-delivery-093000885.html.

[10] "Meituan-Dianping 2020 Interim Report," Webb-site Who's Who. Sep 9, 2020. https:/ /www1. hkexnews. hk/listedco/listconews/sehk/2020/0909/2020090900259.pdf. 